\title{
An Efficient Asynchronous High-Frequency Steady-State Visual Evoked Potential-Based Brain-Computer Interface speller: The Problem of Individual Differences
}

\begin{abstract}
:
Background: Brain-computer interfaces (BCIs) based on steady-state visual evoked potentials (SSVEPs) provide high rates of accuracy and information transfer rate, but need user's attention to flickering visual stimuli. This quickly leads to eye-fatigue when the flickering frequency is in the low-frequency range. High-frequency flickering stimuli $(>30 \mathrm{~Hz})$ have been proposed with significantly lower eye-fatigue. However, SSVEP responses in this frequency range are remarkably weaker, leading to doubts about usability of high-frequency stimuli to develop efficient BCI systems. The purpose of this study was to evaluate if a practical SSVEP Speller can be developed with Repetitive Visual Stimuli in the high-frequency range. Methods: An asynchronous high-frequency $(35-40 \mathrm{~Hz})$ speller for typing in Persian language was developed using five flickering visual stimuli. Least absolute shrinkage and selection operator algorithm with two user-calibrated thresholds was used to detect the user's selections. A total of 14 volunteers evaluated the system in an ordinary office environment to type 9 sentences consist of 81 characters with a multistage virtual keyboard. Results: Despite very high performance of 6.9 chars/min overall typing speed, average accuracy of $98.3 \%$, and information transfer rate of $64.9 \mathrm{bpm}$ for eight of the participants, the other six participants had serious difficulty in spelling with the system and could not complete the typing experiment. Conclusions: The results of this study in accordance with some previous studies suggest that high rate of illiteracy in high-frequency SSVEP-based BCI systems may be a major burden for their practical application.
\end{abstract}

Keywords: Brain-computer interface illiteracy, Brain-computer interface speller, brain-computer interfaces, steady-state visual evoked potentials

\section{Introduction}

Brain-computer interfaces (BCI) based on steady-state visual evoked potentials (SSVEP) have recently gained great attention due to their high accuracies and information transfer rates, low training requirement and fewer electroencephalography (EEG) electrodes for extracting corresponding responses. ${ }^{[1]}$ In these systems, several repetitive visual stimuli (RVS) are placed in front of the user, flickering at different frequencies/phases. When the user focuses at one of these RVSs as available choices, his/her intention is recognized by detecting the selected frequency/phase in the EEG which is recorded from the visual cortex (occipital area). Low-frequency flickering stimuli $(5-15 \mathrm{~Hz})$ have been extensively used in these systems due to their stronger SSVEP responses. ${ }^{[2-7]}$ Cecotti have developed

This is an open access journal, and articles are distributed under the terms of the Creative Commons Attribution-NonCommercial-ShareAlike 4.0 License, which allows others to remix, tweak, and build upon the work non-commercially, as long as appropriate credit is given and the new creations are licensed under the identical terms.

For reprints contact: reprints@medknow.com a spelling system with RVS frequency range of $6-9 \mathrm{~Hz}$ using six channels of EEG. $^{[2]}$ A 27-character keyboard was used in which each letter could be spelled with three selections. Their system achieved an accuracy of $92.3 \%$ and an average spelling speed of $5.5 \mathrm{char} / \mathrm{min}$. Allison et al. have developed a SSVEP-based BCI speller with a 32-character keyboard using 6 EEG channels. Their system has used the frequency range of $13-16 \mathrm{~Hz}$ and achieved an accuracy of $95.8 \%$, information transfer rate of $13 \mathrm{bit} / \mathrm{min}$, and spelling speed between 0.9 and $2.8 \mathrm{char} / \mathrm{min} \cdot{ }^{[8]}$ Chen et al. have developed a synchronous speller using 40 flickers on a liquid crystal display (LCD) monitor that differs both in their frequencies and phases. RVS frequencies have been in the range of $8-15.8 \mathrm{~Hz}$. They reported an outstanding ITR of $4.5 \mathrm{bits} / \mathrm{s}$ for this system. ${ }^{[9]}$

\footnotetext{
How to cite this article: Ajami S, Mahnam A, Behtaj S, Abootalebi V. An efficient asynchronous high-frequency steady-state visual evoked potentialbased brain-computer interface speller: The problem of individual differences. J Med Sign Sens 2018;XX:XX-XX.

Received: April, 2018. Accepted: September, 2018.
}

\section{Saba Ajami', Amin Mahnam ${ }^{1}$, Samane Behtaj', Vahid Abootalebi}

${ }^{1}$ Department of Biomedical Engineering, Faculty of Engineering, University of Isfahan, Isfahan, ${ }^{2}$ Department of Electrical Engineering, Yazd University, Yazd, Iran
Address for correspondence: Dr. Amin Mahnam. Department of Biomedical Engineering, Faculty of Engineering, University of Isfahan, Isfahan, Iran. E-mail:mahnam@eng.ui.ac.ir

Website: www.jmss.mui.ac.ir DOI: 10.4103/jmss.JMSS_19_18 
However, eye fatigue and tearing are quickly result of focusing at low-frequency flickers and sometimes headache which on the one hand, leads to loss of attention and therefore reduces the system's performance, and on the other hand, degrades the user convenience to practically work with the system in real applications. ${ }^{[10]}$ One of the solutions proposed to overcome this problem is to implement higher frequency RVSs which are less perceptible by the visual system. ${ }^{[11]}$ Since mid-frequency flickers (15-25 Hz) may induce an epileptic seizure with higher probability in susceptible users, only RVSs with frequencies above this range should be used. ${ }^{[12]}$ The point is that while the eye-fatigue decreases by increasing the RVS frequency towards and above the critical flicker frequency, the SSVEP responses degrades, and decreases the accuracy of the system. However, several studies have reported successful implementation of high-frequency SSVEP-based BCIs. ${ }^{[9]}$

Usually, in high-frequency SSVEP-based BCIs, the accuracy of systems and the number of people who can work with the system is lower than low-frequency systems. Chen et al. have developed a synchronous speller consisted of 45 RVSs with $0.2 \mathrm{~Hz}$ frequency differences generated on an LCD monitor. ${ }^{[13]}$ Two target frequency sets in the range of $7-15.8 \mathrm{~Hz}$ and $35.6-44.4 \mathrm{~Hz}$ were used in this study, which was recognized from nine selected electrodes. They achieved an average accuracy of $90.2 \%$ and ITR of 82 bits/minute with RVSs in low-frequency range with a processing window of $3 \mathrm{~s}$, but reported accuracy of $88.7 \%$ and ITR of 61 bits/minute in high-frequency range for a processing time window of $4 \mathrm{~s}$. Won et al. ${ }^{[11]}$ have compared the performance of a BCI for spelling with a QWERTY board $^{[14]}$ consisted of $30 \mathrm{RVSs}$ in the frequency ranges of 6-15 and 26-35 Hz. ${ }^{[11]}$ Their system was implemented for synchronous spelling using 15 channels of EEG and achieved an accuracy of $66.5 \%$ for low-frequency RVSs and $68.9 \%$ for high-frequency RVSs. The accuracies have been below $50 \%$ for 7 of 26 participants participated in these experiments. Sakurada et al. compared two frequency sets of 41, 43, and $45 \mathrm{~Hz}$, and 61, 63, and $65 \mathrm{~Hz}$. The higher frequency set leaded to significantly lower eye fatigue. ${ }^{[15]}$ From 12 participants who participated in their experiments, 10 participants were reported successful in controlling the exoskeleton using the frequency range of 61-65 Hz, while the accuracies were below $80 \%$ for the other two participants. In 2013, Diez et al. have used a system with frequency range of $37-40 \mathrm{~Hz}$ with three channels of EEG. From 15 participants in their study, two have been reported as SSVEP-BCI illiterates who could not work with the system. The accuracy and information transfer rate for the rest have been reported as $96.2 \%$ and $44.6 \mathrm{bits} /$ minute respectively. ${ }^{[16]}$

Asynchronous BCIs offer a more natural form of interaction for the user since he/she has not restricted to a specific framework dictated by the computer to send his/her messages. In these systems, however, the idle state, i.e., when the user has no intention to send messages to the computer have to be recognized from the command state automatically. Diez et al. have realized an asynchronous BCI system using a time-threshold. ${ }^{[16]}$ A recognized target has been considered a valid intention of the user if it was recognized in eight consecutive processing windows (equal to $2 \mathrm{~s}$ ). Some researchers have proposed methods based on comparing the absolute or relative feature values with predefined thresholds..$^{[2,17]}$ Merino et al. have proposed a likelihood ratio test based on distributions of canonical correlation analysis (CCA) scores to distinguish the idle state, ${ }^{[18]}$ while Poryzala and Materka have proposed k-means cluster analysis of CCA coefficients to identify commands from each other and the idle state. ${ }^{[19]}$

Performance of an SSVEP-based BCI system is influenced by many parameters including the RVS intensity, frequency, duty cycle, and color, as well as implemented signal processing algorithms. The effect of these parameters has been investigated in many studies. ${ }^{[20-24]}$ However, despite using appropriate parameters in a BCI, substantial variations can be observed in system's performance for different users. While some people can work with a BCI efficiently, some others are not able to work at all, so that BCI illiteracy is now a well-recognized phenomenon in different BCI paradigms ${ }^{[25]}$ including the SSVEP-based BCI systems. ${ }^{[26]}$ Allison et al. have studied the performance of a low-frequency SSVEP BCI system on 106 individuals and demonstrated that there is a negative correlation between the accuracy of the system and the participants age. ${ }^{[8]}$ Moreover, psychological variables are suggested to affect the performance in working with BCIs. ${ }^{[27]}$ Volosyak et al. have compared the performance of an SSVEP-based BCI system on 86 users, when RVSs in the frequency range of $13-16$ or $34-40 \mathrm{~Hz}$ were used. EEG was recorded in eight channels when users looking at four flickering targets $40 \mathrm{~cm}$ from their eyes to control a robot in a labyrinth. Nearly $97.7 \%$ of the users could control the robot with their BCI system when low-frequency flickers were used, but only $65.1 \%$ could do the same with high-frequency flickers. ${ }^{[28]}$ In contrast with the results reported by Diez et al. ${ }^{[16]}$ and Sakurada et al., ${ }^{[15]}$ this result has questioned the usability of high-frequency RVSs for the development of practical BCI systems that can be used by a large portion of people. Moreover, the experiments' protocols used in some of these studies are far from practical conditions. This includes implementing very large $\mathrm{RVSs}^{[15]}$ relatively small distance of the RVSs from the subject's eyes, ${ }^{[15,28]}$ long processing windows, ${ }^{[13]}$ performing experiments in noise-free laboratories, and use of many EEG channels. Short duration of conducted experiments is also not comparable with practical conditions when the user is supposed to work for several minutes and even hours with the system.

The present study aimed to examine the possibility of developing an asynchronous SSVEP-based speller with 
a pragmatic approach using RVSs in the high-frequency range that can work efficiently for a large group of users. High-frequency RVSs are important in real applications to avoid eye-fatigue. The system was aimed to use only a single channel of EEG for minimum setup time and user convenience, and experiments were performed in an ordinary office with usual daylight luminance. The challenge is low amplitude of SSVEPs in the high-frequency range. High rate of error in recognizing target RVSs in an asynchronous paradigm make typing practically impossible for the user. Here, a high-accuracy frequency detection algorithm has been used for the system, and participants were asked to type a relatively long text, so that the performance of the system can be evaluated practically.

\section{Materials and Methods}

A BCI based on steady-state visual evoked potentials was developed using a single channel of EEG and high-frequency RVSs. A wireless EEG recording device (BioAmp 2, Rayan Mindware ${ }^{[29]}$ ) was used, and the signal processing algorithm as well as the interface was implemented in Matlab 2013 (Mathworks). Separate hardware was used to generate flickering stimuli which were placed around the laptop's LCD. The system was calibrated for each participant in a synchronous test, and then he/she used the system for typing several sentences in an asynchronous test.

\section{Experiment set-up}

Five flickering red light-emitting diodes (LEDs) driven by a microcontroller-based circuit were placed at the corners and above at the middle of the 17 " LCD, adjacent to target boxes that were displayed on the LCD [Figure 1]. The distance of the LEDs from the middle of the screen was about $20 \mathrm{~cm}$. Red color was selected due to stronger SSVEP response in comparison to other colors ${ }^{[24]}$ while the luminance of the LED when flickering at high frequency was not annoying. Each LED was in a square frame of $2.5 \mathrm{~cm} \times 2.5 \mathrm{~cm}$ with opaque cover and had $950 \mathrm{Lx}$ light intensity. The distance of the screen from the participant's

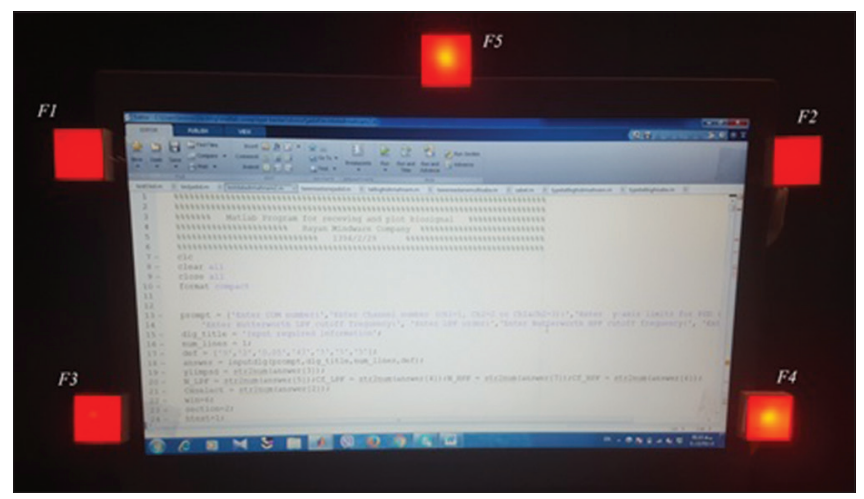

Figure 1: Experiment setup. Five red flickering light-emitting diodes in $2.5 \mathrm{~cm} \times 2.5 \mathrm{~cm}$ opaque cover were placed around a 17-inch monitor. The user's face was about $70 \mathrm{~cm}$ from the middle of the screen eyes was $70 \mathrm{~cm}$, and the flickering frequencies were set to $35,36.2,37.3,38.3$, and $39.4 \mathrm{~Hz}$. This frequency range was used based on previous experiments in our laboratory, to ensure low eye fatigue while having an acceptable signal to noise ratio for SSVEP detection. ${ }^{[30]}$

The wearable EEG recording system used in this study had the capability of recording two channels of EEG with 24-bit resolution and a noise level of $4 \mu \mathrm{V}$. EEG was recorded bipolarly at 250 samples per second from Oz-Pz, and the ground electrode was at $\mathrm{P}_{2}$. Signal quality was assessed by ensuring low electrode impedances, and then by checking the amplitude of $50 \mathrm{~Hz}$ noise, eye-closed alpha activity, and general spectrum of the signal before the experiments.

\section{Processing of recorded electroencephalography}

EEG signal was first passed through fifth-order Butterworth highpass and lowpass filters with cutoff frequencies of 3 and $80 \mathrm{~Hz}$, and a notch filter at $50 \mathrm{~Hz}$. To discriminate between five SSVEP responses, least absolute shrinkage and selection operator (LASSO) model were applied to the filtered EEG. CCA has been mostly used to detect SSVEP responses. ${ }^{[11,32]}$ However, Zhang et al. demonstrated that LASSO estimate ${ }^{[33]}$ provides higher performance for short-time analyzing. ${ }^{[34]}$ In this method, a window of recorded EEG, $y$, is modeled as a linear regression of a design matrix, $\mathrm{X}$ (Eq. 1).

$y=X \beta+\varepsilon$

Here, the design matrix consists of the first two harmonics of symmetric square-waves at stimulus frequencies, and $\varepsilon$ is the noise vector with zero mean and constant variance. LASSO estimate of observation y, is obtained from (Eq. 2):

$\beta=\operatorname{argmin}_{\beta}\left(\|y-X \beta\|_{2}^{2}+\lambda\|\beta\|_{1}\right)$

Where $\lambda$, the penalty parameter, leads to an estimation of $y$ by the minimum number of design components. Eq. 2 can be solved using quadratic programming. ${ }^{[34]}$ Here, the algorithm was implemented using lasso function in MATLAB with NumLambda parameter equal to 2. As considered in this study, $\beta$ includes four components for each target frequency, corresponding to sine and cosine functions at first and second harmonics of that frequency. The absolute values of these components are summed and are named the contribution degree (CD) of that target frequency. In synchronous BCI system, the reference with the highest $\mathrm{CD}$ is considered as the target RVS. However, for an asynchronous BCI system, the algorithm should also discriminate the idle-state; i.e., when the user does not look at any of the RVSs. In general, detection of the idle/control state is based on a predefined threshold, which is related to the amplitude of SSVEP response. However, due to the variability of the EEG signal with time and between individuals, it is difficult to find an optimal threshold to achieve a high true-positive rate (TPR) in the control state while maintaining a low false-positive rate 
in the idle state. ${ }^{[35]}$ In the present study, two thresholds were implemented in the algorithm. A gazed target was recognized by the system only when the corresponding maximum CD was above an "absolute" threshold and its differences with CD s of other RVS frequencies were greater than a second, "difference" threshold. These thresholds are set for each participant based on the results of a calibration test. In a previous study, a significant effect of these two thresholds to improve the accuracy of the system has been demonstrated. ${ }^{[30]}$ In this study, LASSO model was applied to $2 \mathrm{~s}$ time windows with time steps of $0.5 \mathrm{~s}$ (i.e., $75 \%$ overlap). Moreover, the system only confirms the user's focus at a RVS if the corresponding frequency is detected in two consecutive analyzing windows. Considering the $0.5 \mathrm{~s}$ steps for moving the analyzing window forward, this brings only a $0.5 \mathrm{~s}$ delay in the system's response to the user, but significantly reduces the risk of wrong detection of the user's intended frequency.

Considering substantial variation in SSVEP amplitudes for different people, a calibration test was conducted for each participant before the main typing experiment for appropriate selection of absolute and difference thresholds. To determine the thresholds, LASSO model was applied off-line on the EEG signal, and then, by sweeping the "absolute" and "difference" thresholds, their optimum values for highest accuracy was determined. These values were then used in the online typing experiments.

\section{The user interface for spelling}

A multi-stage user interface was developed for spelling in Persian/Arabic language. Figure 2 shows the developed user interface, which includes 32 Persian letters, as well as "space", "question mark", "backspace" and "clear all." The letters are grouped into several boxes. The

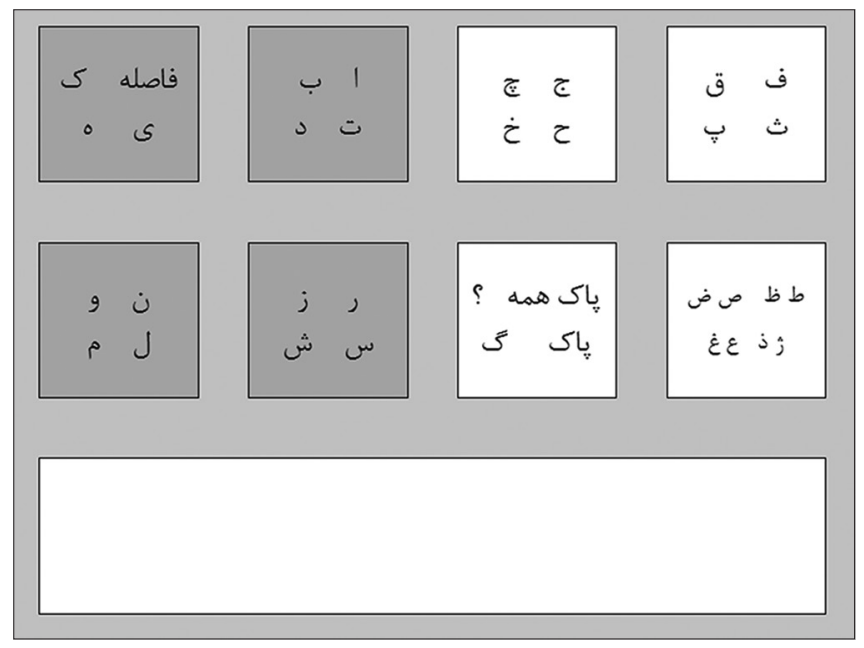

Figure 2: Multistage user interface developed for typing Persian/Arabic letters, using five repetitive visual stimulis. The interface includes 32 Persian letters, "space," "clear," "clear all" and "?." The letters are arranged based on their rate of use in Persian documents, and their similarity. Each letter can be spelled by $2-4$ selections; 16 high-rate letters are spelled with two selections, and eight low-rate letters with 4 selections arrangement of letters in the boxes is based on their rate of use in Persian documents, as calculated in. ${ }^{[36,37]}$ At the same time, similar letters were placed in one box so that they can be easily found by the user. Letters with more rate of use were arranged in left four boxes. Since SSVEP amplitude is higher (on average) at lower frequencies, letters with a higher rate of use were placed in the boxes that correspond to lower flickering frequencies, so that they can be selected more easily. Each of these four boxes [Figure 2] corresponds to one of the RVSs installed at the corners of the screen. The frequency set consisted of $\mathrm{F} 1=36.2, \mathrm{~F} 2=35, \mathrm{~F} 3=37.3, \mathrm{~F} 4=38.3, \mathrm{~F} 5=39.4 \mathrm{~Hz}$. When the user selects one of the boxes by focusing at the corresponding RVS, a second window is opened in which the selected group of letters is distributed in four new boxes and can be selected with focusing again at one of the RVSs. Therefore, the letters that are in the left four boxes of the main screen are selected in only two stages. Letters with lower rate of use were placed in four right boxes. To select these letters, the user has to look at the fifth RVS at the top of the screen, to change the focus from four left boxes to the four right boxes. Then, the letters can be selected in the same way as the letters in the left four boxes. Therefore, these letters need three steps to be selected. The eight least frequently used letters were placed in the right bottom box, and the user have to make one more selection by focusing at one of the left or right RVSs to spell them. As mentioned above, looking at the fifth RVS in the main window leads to change of focus between right and left four boxes. However, looking at this RVS when the second or third selection windows are open closes that window and move the user one step backward in the selection process. This "undo" action is useful when a box has been selected incorrectly by the user or the system.

\section{Evaluation of the system}

A total of 14 healthy individuals (5 males and 9 females; $24 \pm 3.7$ years old) mainly university students participated in this study. All the participants had normal or corrected to normal vision and had no history of visual or neurological disorders. Four of the participants (numbered 3, 4, 5, and 9) had previous experience in working with a BCI system. All the experiments were conducted according to principles described in declaration of Helsinki. ${ }^{[38]} \mathrm{A}$ written informed consent was obtained from each participant before the experiments.

A calibration test was first taken from each participant like the previous study. ${ }^{[30]}$ A sequence of arrows was displayed on the screen as cues to each of the RVSs. Participants looked at each RVS for $5 \mathrm{~s}$ and then looked at the center of the screen where no visual stimulus was present. The test was repeated for each of the RVSs for 7 times in a random order. A bar chart is also presented on the screen demonstrating instantaneous CDs for each RVS obtained from the LASSO model as an online feedback to the user. 
Signals obtained from the calibration test were analyzed offline to appropriately set the "absolute" and "difference" thresholds for the online frequency detection algorithm, as described in Section IIB. The calibration test of each participant was followed by a typing test in which each participant was asked to type the following three sentences for three times.

$$
\text { خارش دست. بول جى شد. غذا كُرم كن. }
$$

The sentences were selected carefully to include most of the letters including least frequently used letters in Persian language. The meaning of sentences is "itching in hand," "what about the money," "heat up some food," and they consisted of 8,9 , and 10 characters, respectively, including the spaces. Therefore, each participant had to type a total of 81 characters in the test. The users were asked to spell all the words correctly and completely. Then the accuracy, selection times, typing speed, and information transfer rate of the system were obtained. Mistakes of the users, however, were not accounted in calculation of the system's accuracy and the typing speed. Moreover, total spelling time of each sentence was measured. Therefore, if due to a system's error, an incorrect selection or spelling was occurred, the time spent to correct it was also taken into account resulting in lower but more realistic value for the typing speed. The same approach was adopted for calculating the system's accuracy: all the selections made by the user including those to correct an error were considered, and the ratio of those selections that were recognized correctly by the system was obtained. With this information, ITR was calculated as defined in: ${ }^{[39]}$

$$
I T R=\left(\log _{2} N+p \times \log _{2} p+(1-p) \times \log _{2}\left(\frac{1-p}{N-1}\right)\right) \cdot R
$$

Where $P$ is the accuracy, $N$ is the number of targets (here 5), and $\mathrm{R}$ is the number of symbols that can be selected in $1 \mathrm{~min}$.

\section{Results}

From 14 participants, six participants could not practically complete the online spelling test, due to either repetitive errors of the system in detecting their intent, or inability to detect SSVEP responses. Frequent repetition of errors in the asynchronous system prevented these users to practically progress in typing the assumed sentences. However, the other eight participants could efficiently perform the spelling task with close to maximum accuracy, and outstanding typing speed. These two groups of participants are named illiterate and literate participants in the next sections.

\section{Calibration test}

Figure 1 demonstrates the two sample screenshots for the calibration test. EEG signals and their power spectral density are presented at left showing that in (a) a good SSVEP response is observed while in (b) the response from another participant can hardly be seen. The right plots preset the corresponding CDs. Table 1 shows the results of the calibration tests on 14 participants, sorted based on obtained accuracies. To calculate the accuracy, the recognized target was considered the one which has maximum $\mathrm{CD}$ provided by LASSO estimate without considering the thresholds. Since there were significant differences in accuracy for different participants, the mean of CDs provided by the LASSO model for target and nontarget frequencies were obtained as well as the spectral power of the SSVEP response and noise at target frequencies for better analysis. EEG power at $\pm 0.3 \mathrm{~Hz}$ intervals around each target frequency was obtained for the periods where each user was looking at each RVS and for the $5 \mathrm{~s}$ periods immediately after that when the user did not look at any of the RVSs.

For participants \#9-14 (illiterate group), the spectral power at target frequencies was close to the noise level, and

Table 1: Variation of average steady-state visual evoked potentials responses and detection accuracies for 14

\begin{tabular}{|c|c|c|c|c|c|c|}
\hline \multicolumn{2}{|c|}{ Participants } & \multirow[t]{2}{*}{ Average accuracy (\%) } & \multicolumn{2}{|c|}{ Average spectral power $\left(\mu V^{2}\right)$} & \multicolumn{2}{|c|}{ Average LASSO coefficients } \\
\hline Number & Gender/age & & Signal & Noise & Target frequency & Nontarget frequencies \\
\hline 1 & Female/24 & 96.3 & $4.9 \pm 1.8$ & $0.28 \pm 0.20$ & $0.12 \pm 0.03$ & $0.03 \pm 0.01$ \\
\hline 2 & Male/26 & 88.3 & $4.8 \pm 2.4$ & $0.38 \pm 0.27$ & $0.11 \pm 0.04$ & $0.03 \pm 0.01$ \\
\hline 3 & Female/26 & 83.9 & $5.2 \pm 2.7$ & $0.43 \pm 0.27$ & $0.10 \pm 0.04$ & $0.04 \pm 0.02$ \\
\hline 4 & Male/13 & 82.1 & $5.3 \pm 3.6$ & $0.21 \pm 0.13$ & $0.09 \pm 0.04$ & $0.03 \pm 0.01$ \\
\hline 5 & Female/28 & 80.3 & $2.6 \pm 0.9$ & $0.27 \pm 0.20$ & $0.08 \pm 0.03$ & $0.03 \pm 0.01$ \\
\hline 6 & Female/24 & 75.1 & $3.2 \pm 1.2$ & $1.2 \pm 0.54$ & $0.11 \pm 0.04$ & $0.06 \pm 0.02$ \\
\hline 7 & Male/23 & 71.1 & $1.3 \pm 0.53$ & $0.24 \pm 0.12$ & $0.06 \pm 0.02$ & $0.03 \pm 0.01$ \\
\hline 8 & Female/27 & 49.1 & $2.1 \pm 1.1$ & $0.68 \pm 0.37$ & $0.09 \pm 0.03$ & $0.06 \pm 0.03$ \\
\hline 9 & Female/28 & 43.1 & $0.52 \pm 0.27$ & $0.55 \pm 0.87$ & $0.03 \pm 0.02$ & $0.02 \pm 0.01$ \\
\hline 10 & Female/22 & 37.7 & $0.1 \pm 0.05$ & $0.08 \pm 0.05$ & $0.01 \pm 0.007$ & $0.01 \pm 0.005$ \\
\hline 11 & Female/24 & 26.3 & $0.68 \pm 0.48$ & $0.4 \pm 0.19$ & $0.05 \pm 0.02$ & $0.05 \pm 0.02$ \\
\hline 12 & Male/25 & 20 & $0.21 \pm 0.23$ & $0.17 \pm 0.1$ & $0.05 \pm 0.02$ & $0.05 \pm 0.02$ \\
\hline 13 & Male/22 & 17.4 & $2.1 \pm 1.1$ & $2.3 \pm 1.1$ & $0.09 \pm 0.03$ & $0.09 \pm 0.03$ \\
\hline 14 & Female/24 & 15.7 & $4.4 \pm 1.8$ & $3.9 \pm 1.4$ & $0.08 \pm 0.04$ & $0.08 \pm 0.04$ \\
\hline
\end{tabular}
participants in the calibration tests

Highlighted rows correspond to participants that could complete the spelling test 
the same can be observed from the CDs. In participants \#9-12, the signal strength is very low, and in participants \#13-14 although the signal strength is appropriate, the noise (background EEG) level is very high, leading to difficulty in recognition of SSVEP responses.

Table 2 provides the mean and standard deviations of relative spectral power of SSVEP responses [by dividing SSVEP to noise power as provided in Table 1], averaged on the trials of literate and illiterate participant groups. These values were lower at higher frequencies except for $39.4 \mathrm{~Hz}$; It is known that the SSVEP response has a local peak at around $40 \mathrm{~Hz}$. Mann-Whitney U-test on SNRs for each target frequency demonstrated that the values are significantly different in literate and illiterate groups for all the target frequencies, rejecting the hypothesis that low SNR at some not all the target frequencies may result in illiteracy.

\section{Spelling experiment}

Only eight participants [top of Table 1] could successfully complete the online spelling experiment. The rest had difficulty in working with the spelling system due to lots of errors occurring in the frequency detection, resulting in either lots of incorrectly identified targets that prevent the user to follow necessary steps to spell the characters, or no identified targets despite several tries of the participant. This can be predicted from significantly low SSVEP response and resulted low accuracies in the calibration test for these participants. Manual adjustment of the thresholds examined in a few participants did not help.

Table 3 demonstrates results from the typing experiment for eight participants who could work with the system. The accuracies are close to the maximum for most of the participants. The total average accuracy was $98.3 \%$ which means only 26 commands of all the participants were identified incorrectly from the total 1552 commands they used to type 648 characters. Note that the accuracy provided here is for the asynchronous spelling system and cannot be compared directly with accuracies provided in Table 1 for synchronous calibration tests. Several factors contribute in this close to maximum accuracy of the asynchronous system, which include applying two thresholds on the CDs, the necessity for detection of a target at two consecutive windows, and prevention of target recognition for up to $2 \mathrm{~s}$ after each selection.

In Table 3, the selection speed and the typing speed are also reported for each participant. The number of selections for spelling characters is a value between 2 and 4 and is not the same for different characters. Therefore, the selection speed is a better measure to evaluate the performance of the signal processing part of the system. The typing speed provides a performance measure of the overall system and is also influenced by the design of the spelling interface. The average selection speed was $15.7 \mathrm{sel} / \mathrm{min}$, and the typing speed was $6.9 \mathrm{char} / \mathrm{min}$ over all the participants. The selection and typing speed values were calculated by dividing the number of selections and characters in each sentence to the total time spent by each participant for typing that sentence. Therefore, these values are also affected by the promptitude of the user in working with the system and the time that is taken by an unfamiliar user to first determine what is the next character to be spelled, second, to find that character in the interface and then, to decide what he/she have to do to type that character.

Most of the participants were completely unexperienced with the spelling system. Therefore, it is expected that these times would reduce as the users practice to work more efficiently, although this requires further experiments with proper statistical analysis. To have a more precise judgment about the system's performance, the response time of the system was obtained during typing one sentence for each participant. The response time was defined as the time taken by the system to detected a user's command from when the user start focusing at each RVS. The results are provided in Table 4 . The average response time of the system for recognizing each selection was $1.98 \mathrm{~s}$, corresponding to $4.4 \mathrm{~s}$ for spelling a letter. The maximum possible selection and typing speed for the proposed system were obtained as 34.5 and 14.6 per minute, if no delay is considered between commands. These are the theoretically maximum values that can be achieved with the system by an ideally perfect user.

\section{Discussion}

In the present study, it was aimed to develop a reliable practical BCI based on high frequency steady-state visual evoked potentials using only a single channel of EEG. A frequency detection algorithm based on LASSO model of SSVEP responses was used in the proposed system. Despite calibration of the system based on the SSVEP responses of each participant, only eight out of 14 participants could efficiently work with the system and complete our experiment of typing a total of 81 characters in 9 short sentences. Performance of the system was quite good for eight BCI-literate users with only $1.98 \mathrm{~s}$ average

Table 2: Average relative spectral power of steady-state visual evoked potentials responses for different target frequencies

\begin{tabular}{lccccc}
\hline Frequency $(\mathrm{Hz})$ & 35 & 36.2 & 37.3 & 38.3 & 39.4 \\
BCI literate group (participants \#1-8) & $12.9 \pm 10.1$ & $10.8 \pm 6.9$ & $9.8 \pm 6.9$ & $8.8 \pm 6.4$ & $16.7 \pm 16.6$ \\
BCI illiterate group (participants \#9-14) & $1.1 \pm 0.43$ & $1.07 \pm 0.71$ & $1.02 \pm 0.3$ & $1.5 \pm 0.46$ & $1.7 \pm 0.54$ \\
\hline
\end{tabular}

BCI - Brain-computer interfaces 
time for each selection, a total typing speed of $6.9 \mathrm{char} / \mathrm{min}$ and a total of 26 errors in detection of the 1552 tried targets selected by the users. The participants stated their satisfaction from the ease of spelling with the system.

Although many studies have been conducted in the field of SSVEP-based BCI systems, there are a few studies which extended their experiments to an actual application of a spelling system. To the best of our knowledge, none of these studies have used high-frequency RVSs in an asynchronous paradigm, and therefore, a comparison between the systems' performances is difficult. The spelling system developed by Cecotti et al. achieved an accuracy of $92.3 \%$ and an average spelling speed of $5.5 \mathrm{char} / \mathrm{min}$ with RVSs in the range of 6-9 Hz. ${ }^{[2]}$ The spelling system developed by Allison et al. achieved an accuracy of $95.8 \%$, information transfer rate of $13 \mathrm{bit} / \mathrm{min}$ and spelling speed between 0.9 and $2.8 \mathrm{char} / \mathrm{min}$ with RVSs in the range of 13-16 Hz. ${ }^{[8]}$ Based on the criteria of being able to type one out of five words for literacy, the team reported a literacy rate of $75.5 \%$ for the above study. ${ }^{[28]}$ However, average SSVEP amplitudes are significantly lower for high-frequency RVSs, and therefore, lower performances are expected for systems in this frequency range. Won et al. have evaluated the performance of a BCI system for synchronous spelling with high-frequency RVSs up to $35 \mathrm{~Hz}$ and achieved an accuracy of $68.9 \%{ }^{[11]}$ while $27 \%$ of participants had accuracies below 50\%. The system

Table 3: Performance characteristics obtained for each "Literate" participant

\begin{tabular}{lccc}
\hline Subject & $\begin{array}{c}\text { Average } \\
\text { accuracy (\%) }\end{array}$ & $\begin{array}{c}\text { Average typing } \\
\text { speed (chars/min) }\end{array}$ & $\begin{array}{c}\text { Average selection } \\
\text { speed (choice/min) }\end{array}$ \\
\hline 1 & 100 & $7.22 \pm 0.85$ & $16.41 \pm 1.71$ \\
2 & 100 & $7.71 \pm 0.76$ & $17.52 \pm 1.23$ \\
3 & 100 & $8.97 \pm 1.07$ & $20.36 \pm 1.76$ \\
4 & 100 & $7.95 \pm 0.56$ & $18.08 \pm 0.73$ \\
5 & $98.60 \pm 2.11$ & $6.67 \pm 0.94$ & $15.15 \pm 2.01$ \\
6 & $97.41 \pm 3.91$ & $6.45 \pm 1.14$ & $14.81 \pm 2.07$ \\
7 & $97.59 \pm 3.36$ & $5.6 \pm 0.88$ & $12.72 \pm 1.45$ \\
8 & $92.78 \pm 6.32$ & $4.51 \pm 1.27$ & $10.32 \pm 2.56$ \\
\hline
\end{tabular}

developed by Chen et al. for synchronous spelling with 45 RVSs achieved an accuracy of $88.7 \%$ and ITR of $61 \mathrm{bits} / \mathrm{min}$ when high-frequency RVSs were used. ${ }^{[13]}$

While the performances cannot directly compared, the asynchronous speller developed in the present study, achieved an accuracy of $98.3 \%$, typing speed of $6.9 \mathrm{char} / \mathrm{min}$ and information transfer rate of $64.9 \mathrm{bit} / \mathrm{min}$ using only a single channel of EEG on literate group. The high performance of the system is believed to be due to application of both amplitude thresholds in the form of "absolute" and 'difference' thresholds on the LASSO CDs, and time threshold so that the system responds only when the same frequency is detected in two consecutive processing windows. Without using these thresholds in the calibration test performed in this study, the average accuracy on all participants were $56.2 \%$ using a single channel of EEG, which is sensible in comparison with $68.9 \%$ accuracy obtained by Won et al. using 15 channels of EEG. ${ }^{[11]}$

Despite the high performance of the proposed system, the illiteracy rate was significant. About $43 \%$ of participant could not finish the asynchronous typing experiment. However, previous studies using high-frequency RVSs have also noted a group of participants that could not work with their systems and the rate is not far from the rates obtained in the current study. Volosyak et al. have reported that only $65.1 \%$ of 86 participants in their study could work with their BCI system for the RVS frequencies in $34-40 \mathrm{~Hz}$ range ${ }^{[28]}$ which is very close to the value obtained in the current study. Note that there are differences in the protocols used in these two studies. RVSs were smaller, and their distance to the eyes was longer in the current study, while the system was tested on each individual after a calibration test. Moreover, participants were faced to a more complicated task of typing 81 characters (186 choices) in a multistage keyboard (compare with only 12 choices in Volosyak et $\left.a l .{ }^{[28]}\right)$. In Won et al., the accuracy of the developed synchronous speller has been below 50\% for 7 of 26 participants. $^{[11]}$ Clearly, such participants cannot practically work with an asynchronous system.

Table 4: The response time of the system for taking a choice, and typing a character, obtained from the moment a participant start focusing to the moment the system respond

\begin{tabular}{lcccc}
\hline Subject & $\begin{array}{c}\text { Character typing time } \\
\text { (sec) }\end{array}$ & $\begin{array}{c}\text { Selection time } \\
\text { (sec) }\end{array}$ & $\begin{array}{c}\text { Corresponding maximum typing speed } \\
\text { (char/min) }\end{array}$ & $\begin{array}{c}\text { Corresponding maximum selection } \\
\text { speed (choice/min) }\end{array}$ \\
\hline 1 & 3.9 & 1.8 & 15.4 & 34.3 \\
2 & 3.1 & 1.4 & 19.6 & 43.6 \\
3 & 3.2 & 1.4 & 19.0 & 42.1 \\
4 & 3.6 & 1.6 & 16.9 & 37.5 \\
5 & 5.7 & 2.6 & 10.5 & 23.3 \\
6 & 4.2 & 1.9 & 14.2 & 31.6 \\
7 & 4.8 & 2.2 & 12.6 & 27.9 \\
8 & 6.8 & 3.1 & 8.9 & 19.7 \\
Average & $4.4 \pm 1.3$ & $1.98 \pm 0.6$ & $14.63 \pm 3.9$ & $32.50 \pm 8.58$ \\
\hline
\end{tabular}


Note that the frequency detection algorithm implemented in this study was designed to reduce false positives of target detection (by applying two thresholds on the CDs and the necessity for detection of a target at two consecutive windows) which leaded to outstanding accuracy of $98.3 \%$. This strategy may lead to some increase in the rate of false negatives, and therefore the rate of illiteracy. On the other hand, it is demonstrated that the optimum electrode position for recording high amplitude SSVEP responses is different for different users. ${ }^{[40]}$ Despite the use of only a single channel of EEG in the present study the illiteracy rate is comparable to the rate in previous studies where several channels have been used (15 channels in Won et al. ${ }^{[11]}$ and 8 channels in Volosyak et al. $\left.{ }^{[28]}\right)$. Multichannel EEG recording is expected to somehow compensate the effect of none-optimum electrodeposition for SSVEP detection by implementing spatial filtering techniques. Despite this, the illiteracy rate is quite high in those studies as well. This can be an important burden in practical applications of SSVEP BCI systems.

From the results of the calibration test, two groups of participants with illiteracy can be observed. In participants \#9-12 the SSVEP responses were one order of magnitude weaker than those for literate group. On the other hand, participants \#13-14 had comparable SSVEP amplitudes; however, the background EEG activity was high so that signal could not be discriminated from that. Figure 3 shows sample power spectral densities of the EEG signal when different participants were looking at target frequency $36.2 \mathrm{~Hz}$ compared to when they were looking at the middle of the screen. The plots are provided for participant \#2 with strong SSVEP response, participant \#7 with relatively weak response but also weaker background EEG activity, as well as for participant \#10 with no SSVEP response and for participant \#14 with relatively high background EEG activity.

In the first group, illiteracy may be occurred due to cancelation of SSVEPs generated from neurons responsible to different parts of the visual field. As explained in Vanegas et al., ${ }^{[41]}$ cruciform geometry of the visual cortex as the main source of SSVEP responses, is such that upper and lower horizontal field octants of visual space project to the floor and ceiling of the contralateral calcarine sulcus, while the upper and lower vertical field octants project to the ventral and dorsal medial surface on the lips of the calcarine sulcus. Due to this specific configuration, the SSVEP responses generated from different parts of the visual field tends to cancel out each other. This cancelation may occur in different degrees in different individuals resulted in significant individual differences in SSVEP responses, especially for high-frequency responses that are inherently lower in amplitude. The cancelation depends somehow on the position of the recording electrode and can describe individual differences in optimum electrodeposition for SSVEP detection. Moreover, as proposed by Vanegas et al., using specifically designed RVSs that provide flickers with carefully adjusted phases for different regions of the visual field may significantly increase the SSVEP response and reduce the rate of illiteracy for high-frequency SSVEP-based BCIs. However, this should be evaluated in a separate study. Besides, SSVEP cancelation may only describe low amplitude of
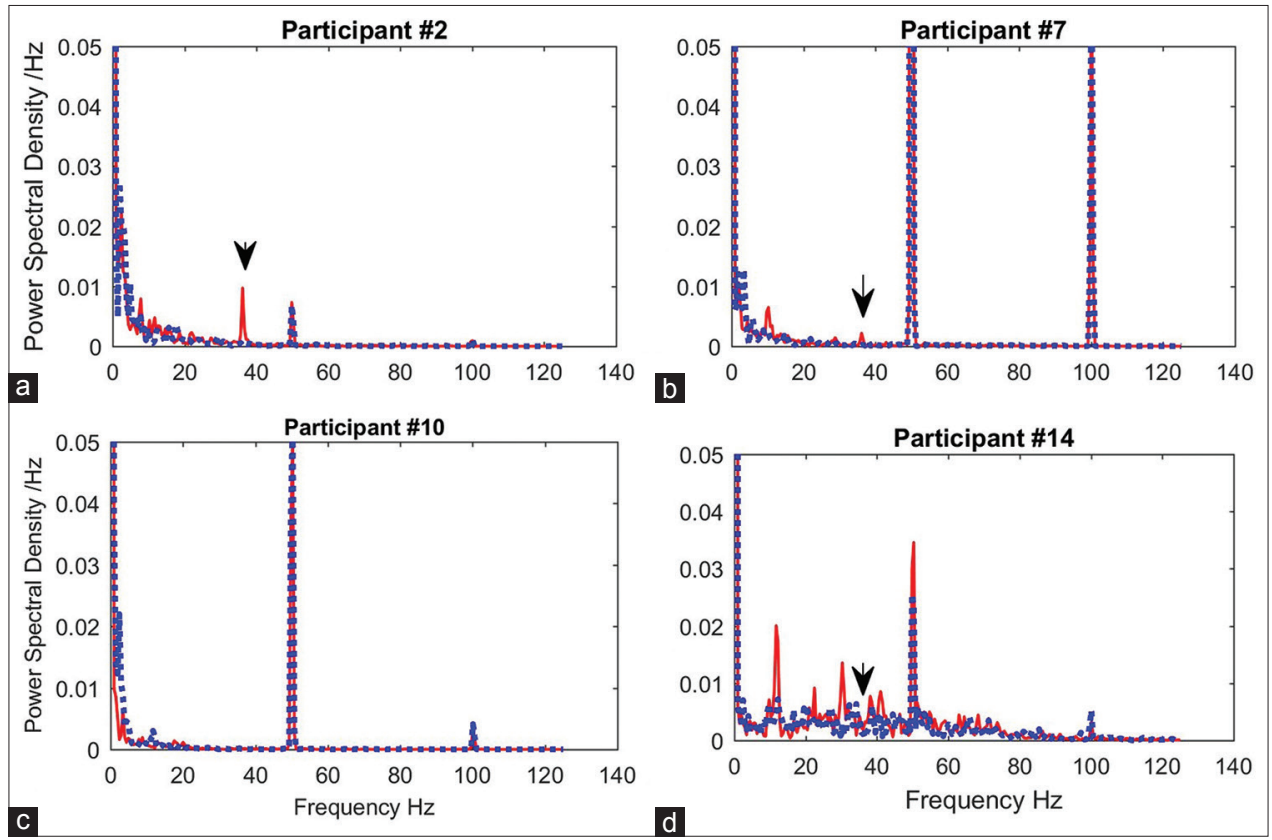

Figure 3: Power spectral density of electroencephalography when participants (a) \#2, (b) \#7, (c) \#10 and (d) \#14 were looking at target frequency of $36.2 \mathrm{~Hz}$ compared with when looking at the middle of the screen. The figures demonstrate a different response and background electroencephalography strengths which made typing possible for participants \#2 and \#7 but not for participants \#10 and \#14 
SSVEP responses, while the problem in the other group of illiterate participants is very high amplitude of background EEG.

\section{Conclusions}

An efficient asynchronous high-frequency SSVEP-based BCI speller was developed in this study using only a single channel of EEG. A total of 14 participants were asked to type 81 characters with this system. Despite outstanding performance of the system for eight participants, others could not type with the system. This high rate of illiteracy was observed although a calibration test was performed for each participant to adjust thresholds in the LASSO-based frequency detection algorithm implemented in this study. The use of only a single channel of EEG which is important for simple use of the system in practical applications may not be the reason for this high illiteracy rate since some other studies that used multiple channels of EEG have also reported similarly high rates of illiteracy. A reason for at least part of this illiteracy may be small amplitude of high-frequency SSVEP responses and specific placement of neurons in the visual cortex so that their responses cancel out each other. It is yet to be studied if specific techniques in visual stimulus design may improve the rate of illiteracy for high-frequency RVSs. However, the outstanding performance of the algorithm used in this study to detect target frequencies could not improve the rate of literacy.

Therefore, BCI illiteracy seems to be a major burden in the practical use of high-frequency SSVEP-based BCIs. Other studies specifically on the reason of this high rate of illiteracy and the ways to decrease it would help in the development of practical SSVEP BCI systems. Moreover, other techniques to reduce eye fatigue in SSVEP BCI systems may be investigated.

\section{Financial support and sponsorship}

Cognitive science and technologies council (Grant No. 78).

\section{Conflicts of interest}

There are no conflicts of interest.

\section{References}

1. Cheng M, Gao X, Gao S, Xu D. Design and implementation of a brain-computer interface with high transfer rates. IEEE Trans Biomed Eng 2002;49:1181-6.

2. Cecotti H. A self-paced and calibration-less SSVEP-based brain-computer interface speller. IEEE Trans Neural Syst Rehabil Eng 2010;18:127-33.

3. Chen X, Wang Y, Zhang S, Gao S, Hu Y, Gao X, et al. A novel stimulation method for multi-class SSVEP-BCI using intermodulation frequencies. J Neural Eng 2017;14:026013.

4. Kwak NS, Müller KR, Lee SW. A convolutional neural network for steady state visual evoked potential classification under ambulatory environment. PLoS One 2017;12:e0172578.

5. Nakanishi M, Wang Y, Wang YT, Mitsukura Y, Jung TP. A high-speed brain speller using steady-state visual evoked potentials. Int J Neural Syst 2014;24:1450019.
6. Ortner R, Allison BZ, Korisek G, Gaggl H, Pfurtscheller G. An SSVEP BCI to control a hand orthosis for persons with tetraplegia. IEEE Trans Neural Syst Rehabil Eng 2011;19:1-5.

7. Trejo LJ, Rosipal R, Matthews B. Brain-computer interfaces for 1-D and 2-D cursor control: Designs using volitional control of the EEG spectrum or steady-state visual evoked potentials. IEEE Trans Neural Syst Rehabil Eng 2006;14:225-9.

8. Allison B, Luth T, Valbuena D, Teymourian A, Volosyak I, Graser A, et al. BCI demographics: How many (and what kinds of) people can use an SSVEP BCI? IEEE Trans Neural Syst Rehabil Eng 2010;18:107-16.

9. Chen X, Wang Y, Nakanishi M, Gao X, Jung TP, Gao S, et al. High-speed spelling with a noninvasive brain-computer interface. Proc Natl Acad Sci U S A 2015;112:E6058-67.

10. Cao T, Wan F, Wong CM, da Cruz JN, Hu Y. Objective evaluation of fatigue by EEG spectral analysis in steady-state visual evoked potential-based brain-computer interfaces. Biomed Eng Online 2014;13:28.

11. Won DO, Hwang HJ, Dähne S, Müller KR, Lee SW. Effect of higher frequency on the classification of steady-state visual evoked potentials. J Neural Eng 2016;13:016014.

12. Pastor MA, Artieda J, Arbizu J, Valencia M, Masdeu JC. Human cerebral activation during steady-state visual-evoked responses. J Neurosci 2003;23:11621-7.

13. Chen X, Chen Z, Gao S, Gao X. A high-ITR SSVEP-based BCI speller. Brain Comput Interfaces 2014;1:181-91.

14. Hwang HJ, Lim JH, Jung YJ, Choi $\mathrm{H}$, Lee SW, Im CH, et al. Development of an SSVEP-based BCI spelling system adopting a QWERTY-style LED keyboard. J Neurosci Methods 2012;208:59-65.

15. Sakurada T, Kawase T, Komatsu T, Kansaku K. Use of high-frequency visual stimuli above the critical flicker frequency in a SSVEP-based BMI. Clin Neurophysiol 2015;126:1972-8.

16. Diez PF, Torres Müller SM, Mut VA, Laciar E, Avila E, Bastos-Filho TF, et al. Commanding a robotic wheelchair with a high-frequency steady-state visual evoked potential based brain-computer interface. Med Eng Phys 2013;35:1155-64.

17. Xia B, Li X, Xie H, Yang W, Li J, He L. Asynchronous brain-computer interface based on steady-state visual-evoked potential. Cogn Comput 2013;5:243-51.

18. Merino LM, Nayak T, Hall G, Pack DJ, Yufei Huang. Detection of control or idle state with a likelihood ratio test in asynchronous SSVEP-based brain-computer interface systems. Conf Proc IEEE Eng Med Biol Soc 2016;2016:1568-71.

19. Poryzala P, Materka A. Cluster analysis of CCA coefficients for robust detection of the asynchronous SSVEPs in brain-computer interfaces. Biomed Signal Process Control 2014;10:201-8.

20. Duszyk A, Bierzyńska M, Radzikowska Z, Milanowski P, Kuś R, Suffczyński P, et al. Towards an optimization of stimulus parameters for brain-computer interfaces based on steady state visual evoked potentials. PLoS One 2014;9:e112099.

21. Gollee H, Volosyak I, McLachlan AJ, Hunt KJ, Gräser A. An SSVEP-based brain-computer interface for the control of functional electrical stimulation. IEEE Trans Biomed Eng 2010;57:1847-55.

22. Herrmann CS. Human EEG responses to $1-100 \mathrm{hz}$ flicker: Resonance phenomena in visual cortex and their potential correlation to cognitive phenomena. Exp Brain Res 2001; 137:346-53.

23. Shu X, Yao L, Meng J, Sheng X, Zhu X. Visual Stimulus Background Effects on SSVEP-Based Brain-Computer Interface. In $6^{\text {th }}$ International Conference on Intelligent Robotics and Applications (ICIRA). Proceedings, Part. I. Busan, South Korea: 
Springer; 2013. p. 453-62.

24. Tello RJ, Müller SM, Ferreira A, Bastos TF. Comparison of the influence of stimuli color on steady-state visual evoked potentials. Res Biomed Eng 2015;31:218-31.

25. Blankertz B, Losch F, Krauledat M, Dornhege G, Curio G, Müller KR, et al. The berlin brain - Computer interface: Accurate performance from first-session in BCI-naïve subjects. IEEE Trans Biomed Eng 2008;55:2452-62.

26. Allison BZ, Neuper C. Could anyone use a BCI? in brain-computer interfaces. In: Tan D, Nijholt A, editors. Human-Computer Interaction Series. London: Springer; 2010. p. 35-54.

27. Nijboer F, Furdea A, Gunst I, Mellinger J, McFarland DJ, Birbaumer $\mathrm{N}$, et al. An auditory brain-computer interface (BCI). J Neurosci Methods 2008;167:43-50.

28. Volosyak I, Valbuena D, Lüth T, Malechka T, Gräser A. BCI demographics II: How many (and what kinds of) people can use a high-frequency SSVEP BCI? IEEE Trans Neural Syst Rehabil Eng 2011;19:232-9.

29. Available from: http://www.rayanware.com.[Last accessed on 2018 Jan 05].

30. Ajami S, Mahnam A, Abootalebi V. Development of a practical high frequency brain-computer interface based on steady-state visual evoked potentials using a single channel of EEG. Biocybern Biomed Eng 2018;38:106-14.

31. Lin Z, Zhang $\mathrm{C}, \mathrm{Wu} \mathrm{W}$, Gao X. Frequency recognition based on canonical correlation analysis for SSVEP-based BCIs. IEEE Trans Biomed Eng 2006;53:2610-4.

32. Zhang $\mathrm{Y}$, Zhou G, Jin J, Wang $\mathrm{X}$, Cichocki A. Frequency recognition in SSVEP-based BCI using multiset canonical correlation analysis. Int J Neural Syst 2014;24:1450013.

33. Tibshirani R. Regression shrinkage and selection via the lasso. J R Stat Soc Series B Stat Methodol 1996;58:267-88.

34. Zhang Y, Jin J, Qing X, Wang B, Wang X. LASSO based stimulus frequency recognition model for SSVEP BCIs. Biomed Signal Process Control 2012;7:104-11.

35. Pan J, Li Y, Zhang R, Gu Z, Li F. Discrimination between control and idle states in asynchronous SSVEP-based brain switches: A pseudo-key-based approach. IEEE Trans Neural Syst Rehabil Eng 2013;21:435-43.

36. Rajae H. Determination of the Frequent Words in Persian Language News Media to Educate Non-Persian Speakers. MSc Thesis. Payam Noor University; 2010.

37. Mansoori M. Investigation of frequency of characters in Persian language and their place on computer keyboards. J Linguistics Khorasan Dialects 2013;4:109-27.

38. General Assembly of the World Medical Association. World medical association declaration of helsinki: Ethical principles for medical research involving human subjects. J Am Coll Dent 2014;81:14-8.

39. Wolpaw JR, Ramoser H, McFarland DJ, Pfurtscheller G. EEG-based communication: Improved accuracy by response verification. IEEE Trans Rehabil Eng 1998;6:326-33.

40. Wang Y, Zhang Z, Gao X, Gao S. Lead selection for SSVEP-based brain-computer interface. Conf Proc IEEE Eng Med Biol Soc 2004;6:4507-10.

41. Vanegas MI, Blangero A, Kelly SP. Exploiting individual primary visual cortex geometry to boost steady state visual evoked potentials. J Neural Eng 2013;10:036003.

\section{BIOGRAPHIES}

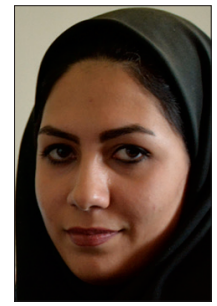

Saba Ajami received her BSc in Electrical Engineering from Yazd University and her $\mathrm{MSc}$ in Biomedical Engineering from University of Isfahan. She then continued her work as a researcher at Isfahan NeuroTechnology Lab for two years. Her research interests are EEG signal processing mainly in the context of Brain Computer Interfaces.

Email: saba_ajami2000@yahoo.com

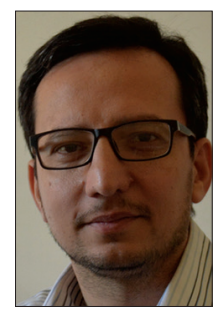

Amin Mahnam is associate professor of Biomedical Engineering at University of Isfahan and head of Isfahan Neuro-Technology Lab. He has BSc degree in Electrical Engineering from Sharif University of Technology and MSc and PhD degrees in Biomedical Engineering from Amirkabir University of Technology. His research interest is Brain-Computer Interfaces and Biomedical Instrumentation.

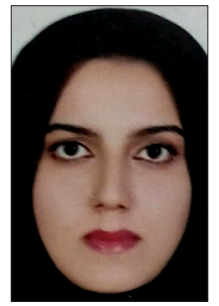

Samane Behtaj received her BSc and MSc in Biomedical Engineering from University of Isfahan. She is now a co-founder of "Rayan Mindware", a knowledge-based start-up company at Isfahan Science and Technology Town, with interest in development of biomedical instruments.

Email: samanebehtaj@gmail.com

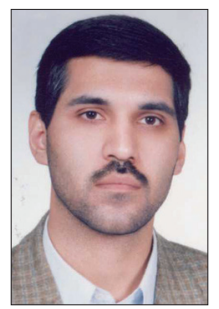

Vahid Abootalebi received the B.S. and M.S. degrees in electrical Engineering from Sharif University of Technology, Tehran, Iran, in 1997 and 2000, respectively. He also received his Ph.D. degree in biomedical engineering from Amirkabir University of Technology, Tehran, Iran in 2006. Since 2007, he has been working as a faculty member of the Electrical Eng. Department of Yazd University, where he is currently an Associate Professor. His main research interests include biomedical signal processing and pattern recognition.

Email: abootalebi@yazd.ac.ir 